\title{
Preferentially enhanced gene expression from a synthetic human telomerase reverse transcriptase promoter in human cancer cells
}

\author{
SUNG JIN KIM ${ }^{1}$, HAN SAEM LEE ${ }^{2}$, JUNE HO SHIN ${ }^{4}$, CHUL GEUN KIM $^{4}$, SUNJOO JEONG $^{5}$ \\ KEERANG PARK ${ }^{6}$, HAN CHOE $^{2,3}$ and HEUIRAN LEE ${ }^{1,3}$

\begin{abstract}
Departments of ${ }^{1}$ Microbiology and ${ }^{2}$ Physiology, ${ }^{3}$ Research Institute for Biomacromolecules, University of Ulsan College of Medicine; ${ }^{4}$ Department of Life Science and Research Institute of Natural Sciences, College of Natural Sciences, Hanyang University, ${ }^{5}$ Department of Molecular Biology, College of Natural Sciences, Dankook University, Seoul;

${ }^{6}$ Department of Biotechnology, Juseong University, Chung-Buk, Korea
\end{abstract}

Received April 18, 2006; Accepted July 17, 2006

\begin{abstract}
Although the human telomerase reverse transcriptase (hTERT) promoter can regulate cancer-specific genes, it is generally too weak to be effective. We therefore attempted to improve the potency of synthetic hTERT promoters by fusing the core element $(\mathrm{E})$ of the hTERT promoter $(\mathrm{H})$ and the tripartite leader sequence $(\mathrm{T})$ from human adenovirus 5 in a combinatorial manner. To determine the potential as cancerspecific promoters, we measured luciferase activity driven by the chimeric hTERT promoters in human cancer cells. Among various constructs, the E3-H-T promoter induced the strongest luciferase activity in all the tested cancer cells. SK-Hep1 and Hela cells experienced 1000- and 11-fold higher expression than the basic hTERT promoter, respectively. Relative to the SV40 universal promoter, the E3-H-T promoter led to higher levels of gene expression. Using EMSA, we found that the hTERT enhancer region was specifically bound to c-Myc and Sp1. Thus, the data suggest that the E3-H-T promoter with up-regulated cancer-specific gene expression could be useful in cancer gene therapy.
\end{abstract}

\section{Introduction}

The selective and efficient expression of therapeutic genes is critical for a satisfactory clinical outcome in cancer gene therapy (1-3). Many studies have evaluated the potential of cancer-specific gene regulatory elements (4). For example, a prostate-specific element seems practical for inducing prostate cancer-specific gene expression $(5,6)$. A carcinoembryonic antigen (CEA) gene promoter can be utilized to regulate genes targeted for colorectal and lung cancer $(7,8)$. MUC-1 and E2F

Correspondence to: Dr Heuiran Lee, Department of Microbiology, University of Ulsan College of Medicine, 138-736 Seoul, Korea

E-mail: heuiran@amc.seoul.kr

Key words: hTERT promoter, c-Myc, Sp1, gene regulatory element, TPL sequence, cancer gene therapy promoters have been applied to breast cancers and to cancers defective in the RB gene, respectively $(9,10)$. However, the specificity of these promoters is restricted to certain tumor types due to the association of gene regulatory components with specific human cancers.

Human telomerase reverse transcriptase (hTERT), the catalytic subunit of telomerase, is up-regulated in more than $80 \%$ of human cancers (11). Therefore, the hTERT promoter may be an attractive candidate for wide coverage of cancerspecific gene expression $(12,13)$. The cancer-specific potential of the hTERT promoter has been demonstrated by introducing various therapeutic genes under the control of the hTERT promoter, including genes encoding Bax, p53 and caspase- 8 (14-21). The unmodified hTERT promoter, however, is generally too weak to induce sufficient gene expression $(17,18)$. The hTERT promoter has been cloned, and proximal core promoter sequences essential for transcriptional activation have been identified $(14,15)$. Promoter sequencing and functional analysis revealed that a 181-bp core promoter encodes multiple binding motifs for Sp1 and c-Myc as part of its gene regulatory function. The subgroup $\mathrm{C}$ human adenovirus tripartite leader (TPL) sequence has been shown to enhance gene expression at the translational level $(34,35)$. TPL is about 200 bp in length and greatly facilitates adenoviral late gene expression (26).

To selectively enhance the ability of the hTERT basic promoter to regulate cancer-specific gene expression, we fused the hTERT core promoter sequence (nt -200 to nt -11) and TPL to the hTERT basic promoter. We then investigated the ability of this promoter for regulating gene expression in a variety of human cancer cells. The data indicate that addition of the hTERT element and TPL significantly improves the ability of the hTERT basic promoter to regulate cancer-specific gene expression.

\section{Materials and methods}

Cell lines. Human breast (MCF-7), liver (SK-Hep1, HepG2 and Hep3B), and cervical (HeLa and 293) cancer cell lines were purchased from the American Type Culture Collection (ATCC; Manassas, VA). The 911 cell line from human 

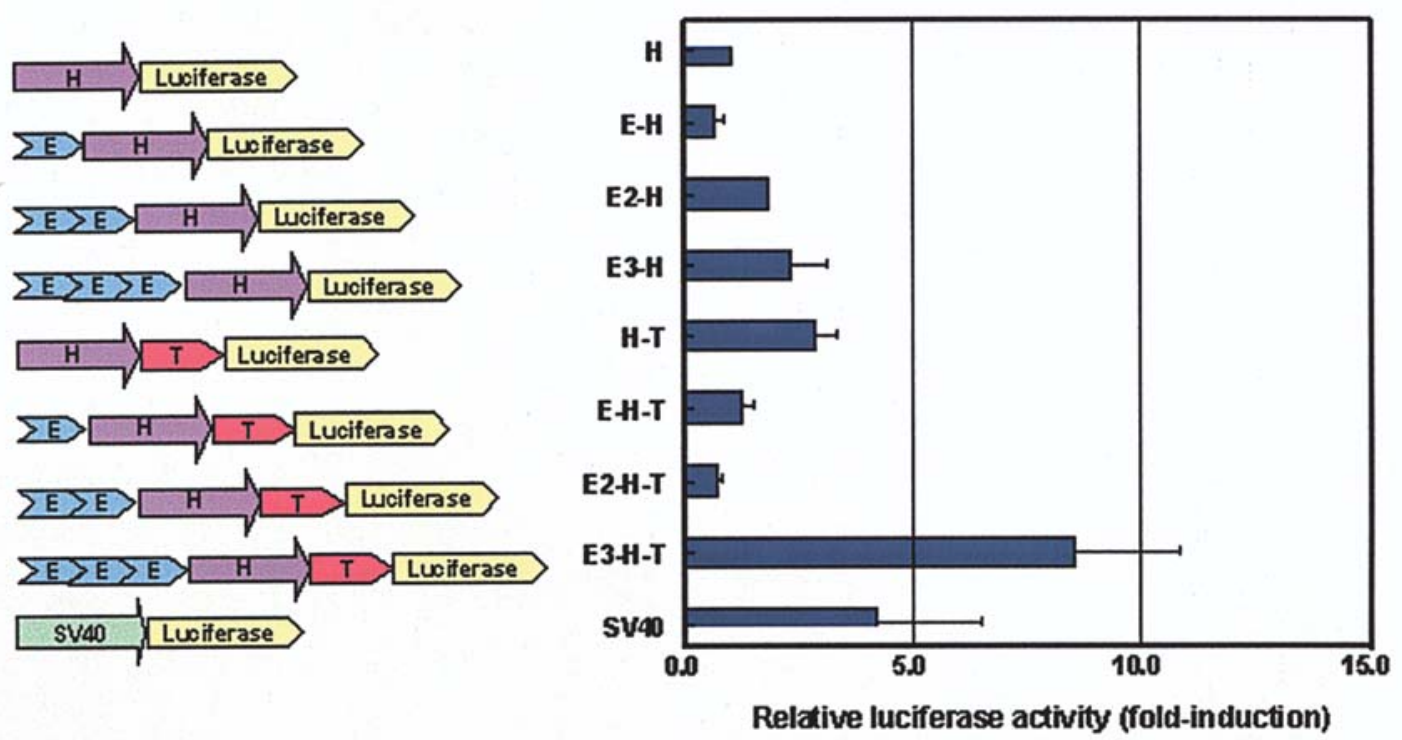

Figure 1. Relative luciferase activity driven by variously modified hTERT promoters in MCF-7 cells. The pGL2-based expression vectors containing luciferase controlled by various hTERT promoters were constructed as described in Materials and methods (left). MCF-7 cells were transiently transfected with each construct, as well as with pGKßgal plasmid. Forty-eight hours later, cell lysates were prepared, and their luciferase activities were normalized relative to $\beta$-galactosidase activity. The relative luciferase activity in each cell line was compared with that of the hTERT basic construct. H, hTERT basic promoter; E, regulatory region of the hTERT promoter; T, TPL sequence; SV40, SV40 promoter.

embryonic retinoblasts, was the generous gift by Dr Van der Eb (University of Leiden, The Netherlands) and the thyroid cancer cells have been described previously (25). Cells were maintained in Dulbecco's modified Eagle's medium supplemented with $10 \%$ fetal bovine serum, L-glutamine $(2 \mathrm{mM})$, penicillin $(100 \mathrm{IU} / \mathrm{ml})$ and streptomycin $(50 \mu \mathrm{g} / \mathrm{ml})$. The cells were incubated in humidified $5 \% \mathrm{CO}_{2} / 95 \%$ air at $37^{\circ} \mathrm{C}$.

Construction of luciferase expression plasmids controlled by modified hTERT promoters. The plasmid pT7-blue-hTERT, encoding the hTERT basic promoter, has been described previously (26). This plasmid, and pGL2-C, were digested with KpnI and HindIII and the hTERT basic promoter was ligated into the pGL2-C vector to generate pGL2-H, in which luciferase was expressed under the control of the hTERT promoter. The hTERT core promoter region (E; nt -200 to nt -11) was PCR-amplified using pT7-blue-hTERT as a template and the primers 5'-GATTCGCGGGCACAGACGC-3' (forward) and 5'-CGCGGAAAGGAAGGGGAGG-3' (reverse). The PCR product was subcloned into pMOSblue (pMOSblue blunt-ending kit; Amersham Pharmacia) and its sequence was verified using an ABI PRISM 377 automatic DNA sequencer. The E piece from pMOSblue-E was digested with HindIII, blunt ended, and digested with KpnI and subcloned into pGL2-H, which had been digested with EcoRI, blunt ended, and digested with KpnI, to generate pGL2-E-H. The TPL sequence (T) was recovered by RT-PCR, using total-RNA from 293 cells infected with human adenovirus 5 as a template, and the primers 5'-ACTCTCTTCCGCATCGCTTGC-3' (forward) and 5'-CTTGCGACTGTGACTGGTTAGAC GCC-3' (reverse), as described previously (27). The PCR product was directly subcloned into pCR2.1 plasmid (Invitrogen, Carlsbad, CA) and its sequence was verified.
The $\mathrm{T}$ fragment isolated from pCR2.1-T was digested with $X b a \mathrm{I}$ and PstI, and subcloned into pSP72-H containing the basic promoter within the BamHI site, which had been digested with the same enzymes. The resultant pSP72-H-T was utilized for further construction of other TERT-based promoters, along with pCR2.1-C, pCR2.1-H and pMOSblue-E.

Luciferase reporter gene assay. Luciferase assay was performed as previously described (28). Briefly, cells in 6-well plates were transfected with $0.8 \mu \mathrm{g}$ of pGL2 construct and $0.2 \mu \mathrm{g}$ of pGKßgal, expressing B-galactosidase from a phosphoglucokinase promoter, by using Lipofectamine plus reagent (Gibco BRL, Gaithersburg, MD). Two days after transfection, the cells were washed with phosphate-buffered saline and lysed in $200 \mu 1$ of reporter lysis buffer (Luciferase assay kit; Promega, Madison, WI). Luciferase activity was determined with a Luminometer, as described by the manufacturer. Values were normalized relative to $\beta$-galactosidase activity. The data were obtained from two or three independent experiments.

Electrophoretic mobility shift assay (EMSA). The $110 \mathrm{bp}$ SmaI subfragment of the E3-H-T promoter, containing the E-box and SP1 binding sites, was 5'-end labeled using $\left[\gamma_{-}{ }^{32} \mathrm{P}\right]-$ ATP and T4 polynucleotide kinase (Promega). For c-Myc and $\mathrm{Sp} 1,1 \times 10^{7} 293 \mathrm{~T}$ cells were harvested and lysed to recover nuclear extracts. One nanogram of the radiolabeled hTERT probe was incubated with $12 \mu \mathrm{l}$ of nuclear extracts at room temperature for $20 \mathrm{~min}$ in a reaction volume of $25 \mu 1$ (31). Two microgrames of the non-specific competitor poly $(\mathrm{dI} \bullet \mathrm{dC})$ was included to minimize the binding of nonspecific proteins to the labeled target DNA. For competition experiments, $1 \mu \mathrm{g}$ of c-Myc or Sp1 antisera (Santa Cruz Biotechnology) was added to the reaction mixture before or after treatment. The mixtures were resolved in $5 \%$ non- 


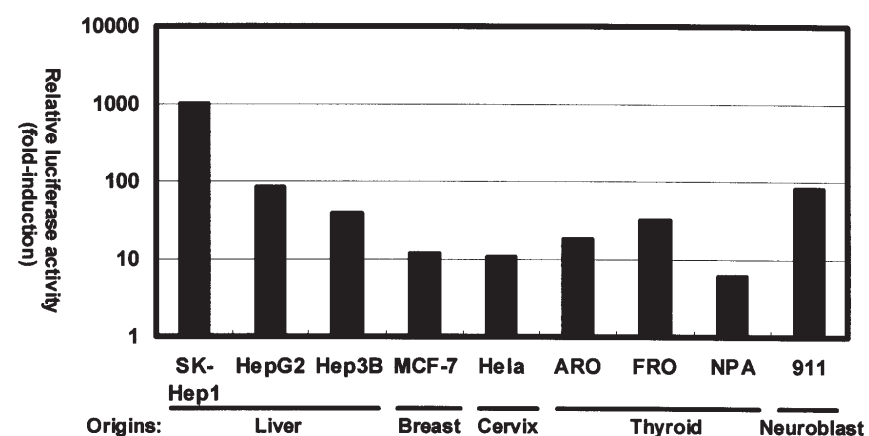

Figure 2. Enhancement of gene expression potency by E3-H-T promoter in various human cancer cells. Relative luciferase activities were determined in various human cancer cell lines, as described in Fig. 1. Luciferase activities driven by E3-H-T were significantly increased in all the human cancer cells. All luciferase activities were normalized relative to that induced by the hTERT basic promoter, which was set to 1.0 in each cell line.

denaturing polyacrylamide gels (60:1 acrylamide to bisacrylamide ratio) containing $2.5 \%$ glycerol in $0.5 \mathrm{X}$ TBE buffer at $4^{\circ} \mathrm{C}$. The gels were dried and visualized by autoradiography.

\section{Results}

Construction of synthetic hTERT promoters and evaluation of their potency in MCF-7 cells. To determine if modified hTERT promoters could preferentially enhance gene expression in human cancer cells, we manufactured various types of hTERT promoters. The hTERT regulatory portion (E) and the TPL sequence $(\mathrm{T})$ were attached to the upstream and downstream of the hTERT basic promoter, respectively $(\mathrm{H}$; Fig. 1, left panel). We then assayed the ability of each construct to regulate luciferase activity in human breast cancer MCF-7 cells. Addition of E-H slightly increased luciferase expression (Fig. 1, right panel). By contrast, the addition of TPL to $\mathrm{H}$ (H-T) substantially enhanced the promoter activity, up to $2.8 \pm 0.5$-fold. Surprisingly, addition of 1-2 E fragments to $\mathrm{H}-\mathrm{T}$ had the opposite effect, decreasing promoter strength, whereas addition of $3 \mathrm{E}$ fragments to $\mathrm{H}-\mathrm{T}$ enhanced luciferase activity by $8.5 \pm 2.3$-fold. Moreover, E3-H-T promoter strength was stronger than that of the SV40 promoter (4.2 \pm 2.3 -fold). A similar pattern of promoter activity was observed in the human HeLa cervical cancer cell line (data not shown). These findings indicate that the triple addition of the regulatory element of hTERT promoter, plus the tripartite leader sequence most dramatically enhance the gene expression potential of the hTERT basic promoter.

Gene expression ability of the synthetic E3-H-T promoter in various human cancer cells. We assayed whether the E3-H-T promoter would up-regulate gene expression in other human cancer cell lines. Hepatocellular carcinoma (SK-Hep1 and Hep3B), hepatoblastoma (HepG2), undifferentiated thyroid (ARA), follicular (FRO), poorly differentiated papillary carcinoma (PNA), and retinoblastoma (911) cells were employed (Fig. 2) (25). As expected, we found that the newly synthesized E3-H-T promoter could markedly enhance luciferase activity, compared with the hTERT basic promoter. The highest increase

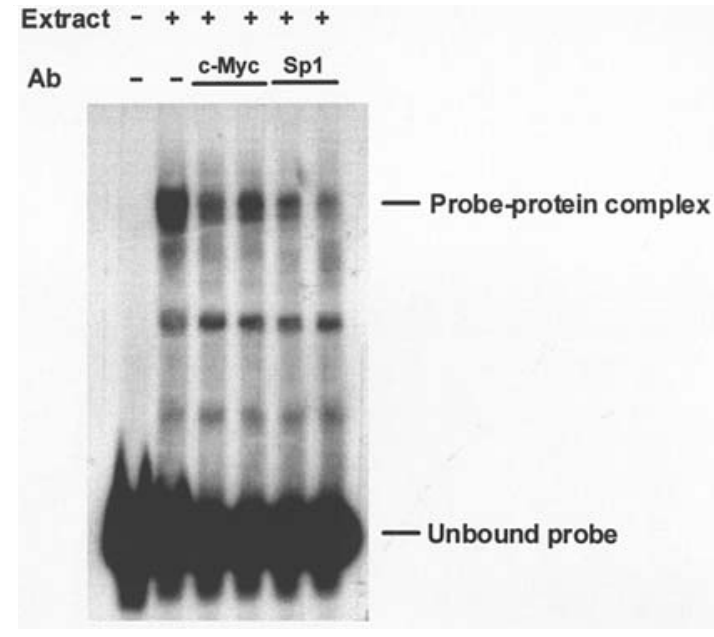

Figure 3. Specific binding of c-Myc and Sp1 to the hTERT promoter. The ability of c-Myc and Sp1 to bind to the E3-H-T promoter was analyzed by incubating cell extracts containing c-Myc and $\mathrm{Sp} 1$ with a radioactive probe covering the putative binding motifs. The reaction mixtures were resolved by running $6 \%$ non-reducing native PAGE and exposing the gels to X-ray film. A significantly retarded band was clearly observed only in the absence of competitors. Lane 1, DNA probe with no cell extract (negative control); lane 2, probe plus cell extract; lanes 3 and 4, probe plus cell extract plus c-Myc antiserum; lanes 5 and 6, probe plus cell extract plus Sp1 antiserum.

was observed in SK-Hep1 cells (1000-fold), whereas the lowest was observed in HeLa cells (11-fold).

c-Myc and Spl binding to the E3-H-T promoter. We used EMSA assays to determine whether the c-Myc and Sp1 transcription factors directly interact with their presumptive binding sites in the E region of E3-H-T (Fig. 3). Radioactive DNA probes covering the c-Myc and Sp1 binding motifs using E3-H-T was synthesized as a template. c-Myc and Sp1 were directly obtained from $293 \mathrm{~T}$ nuclear extracts. We observed that both c-Myc and Sp1 were associated with the E region, as revealed by retardation of the labeled band (lane 2). Moreover, the addition of antibodies to c-Myc and Sp1 antibody efficiently inhibited this complex, indicating specific interactions between the target site and the transcription factors (lanes 3-6). These findings indicate that endogenous c-Myc and $\mathrm{Sp} 1$ in human cancer cells could specifically bind to binding motifs in the E3-H-T promoter, resulting in further activation of the synthetic promoter.

\section{Discussion}

The human telomerase reverse transcriptase (hTERT) promoter holds promising potential for a wide range of cancer-targeted gene expression. One of the major limitation of the hTERT basic promoter, however, is the relatively low level of gene expression (our data, 17,29). To overcome this drawback, we generated a genetically modified hTERT promoter (E3-H-T), leading to improved gene expression.

The hTERT promoter has been identified to lack both a TATA and a CAAT box, whereas the hTERT core region (nt -181 to $n t+77$ ) includes two distal and proximal E-boxes for c-Myc and internal five Sp1 binding sites. Availability of these transcription factors provides strong evidence for their 
direct involvement in hTERT activation (14,31). Previously, various synthetic hTERT promoters were generated by inserting gene regulatory elements originating from either hTERT or other sources, including TATA boxes $(20,26)$, c-Myc binding sites (E-boxes) or Sp1 sites $(21,32,33)$. The data clearly showed that preferential gene expression can be driven by these kinds of modification. Addition of copies of c-Myc and Sp1 binding sites to the original hTERT promoter was shown to improve cancer-selective amplification of the conditionallyreplicating recombinant adenovirus (32). Taken together with our data, the findings strongly indicate that multimerization of the hTERT core region could increase the gene expression by hTERT in a cancer-specific manner. Our finding also implies that the up-regulated gene expression occurs via the direct interaction between transcription factors and presumptive binding motifs. However, the promoter strength, even with the triple addition of the hTERT core region (E3-H), was not as strong as expected.

The TPL motif has been found to positively prompt gene expression at the translational level, by stimulating mRNA export from the nucleus to the cytoplasm and by no need for translational factors $(34,35)$. Based on this, we attached a TPL sequence, along with the hTERT core region, to the basic promoter. We found that the addition of TPL and three transcription factor-binding motifs (E3-H-T) dramatically increased gene expression. Activation of the $\mathrm{E} 3-\mathrm{H}-\mathrm{T}$ promoter was observed exclusively in cancer cells, not in normal human cells, such as the human lung fibroblast cell line MRC-5 (data not shown). Interestingly, the insertion of the transcription factor-binding motif in the opposite direction downregulated promoter activity to $0.03-0.1$ of that in its absence (data not shown). This may have been caused by an inappropriate three-dimensional space created by the opposite orientation of the binding motif. We also found that the increased expression by E3-H-T varied widely in the different cancer cells, which may have been due to a variation in the abundance of endogenous transcription factors.

In summary, we found that the novel synthetic hTERT promoter, E3-H-T, significantly enhanced gene expression. This promoter may have practical potential in cancer gene therapy, and we are currently investigating this issue by testing its ability to enhance the expression of therapeutic genes, such as the anti-angiogenesis gene.

\section{Acknowledgements}

We thank J.Y. Whang for critical reading of the manuscript. This study was supported by grants from the Ministry of Science and Technology M10534050002-05N3405-00200, H.L. and M10534040005-05N3404-00511, K.P.), Seoul, Korea.

\section{References}

1. Vile RG and Rus NR: Cancer gene therapy: hard lessons and new courses. Gene Ther 7: 2-8, 2000.

2. Paillard F: Cancer gene therapy annual conference 1997: trends and news. Hum Gene Ther 9: 283-286, 1998.

3. Marchisone C, Pfeffer U, Del Grosso F, Noonan DM, Santi L and Albini A: Progress towards gene therapy for cancer. J Exp Clin Cancer Res 19: 261-270, 2000.
4. Harrington KJ, Linardakis E and Vile RG: Transcriptional control: an essential component of cancer gene therapy strategies? Adv Drug Deliv Rev 44: 167-184, 2000.

5. Choo R, De Boer G, Klotz L, et al: PSA doubling time of prostate carcinoma managed with watchful observation alone. Int $\mathbf{J}$ Radiat Oncol Biol Phys 50: 615-620, 2001.

6. Rodriguez R, Schuur ER, Lim HY, Henderson GA, Simons JW and Henderson DR: Prostate attenuated replication competent adenovirus (ARCA) CN706: a selective cytotoxic for prostatespecific antigen-positive prostate cancer cells. Cancer Res 57: 2559-2563, 1997.

7. Osaki T, Tanio Y, Tachibana I, et al: Gene therapy for carcinoembryonic antigen-producing human lung cancer cells by cell type-specific expression of herpes simplex virus thymidine kinase gene. Cancer Res 54: 5258-5261, 1994.

8. Kijima T, Osaki T, Nishino K, et al: Application of the Cre recombinase/loxP system further enhances antitumor effects in cell type-specific gene therapy against carcinoembryonic antigen-producing cancer. Cancer Res 59: 4906-4911, 1999.

9. Kurihara T, Brough DE, Kovesdi I and Kufe DW: Selectivity of a replication-competent adenovirus for human breast carcinoma cells expressing the MUC1 antigen. J Clin Invest 106: 763-771, 2000.

10. Putzer BM, Rumpf H, Rega S, Brockmann D and Esche H: E1A $12 \mathrm{~S}$ and $13 \mathrm{~S}$ of the transformation-defective adenovirus type 12 strain CS-1 inactivate proteins of the RB family, permitting transactivation of the E2F-dependent promoter. J Virol 71: 9538-9548, 1997

11. Kim NW, Piatyszek MA, Prowse KR, et al: Specific association of human telomerase activity with immortal cells and cancer. Science 266: 2011-2015, 1994.

12. Shay JW and Bacchetti S: A survey of telomerase activity in human cancer. Eur J Cancer 33: 787-791, 1997.

13. Kyo S, Takakura M and Inoue M: Telomerase activity in cancer as a diagnostic and therapeutic target. Histol Histopathol 15: 813-824, 2000.

14. Koga S, Hirohata S, Kondo Y, et al: A novel telomerasespecific gene therapy: gene transfer of caspase- 8 utilizing the human telomerase catalytic subunit gene promoter. Hum Gene Ther 11: 1397-1406, 2000.

15. Gu J, Kagawa S, Takakura M, et al: Tumor-specific transgene expression from the human telomerase reverse transcriptase promoter enables targeting of the therapeutic effects of the Bax gene to cancers. Cancer Res 60: 5359-5364, 2000 .

16. Komata T, Koga S, Hirohata S, et al: A novel treatment of human malignant gliomas in vitro and in vivo: FADD gene transfer under the control of the human telomerase reverse transcriptase gene promoter. Int J Oncol 19: 1015-1020, 2001.

17. Kanaya T, Kyo S, Hamada K, et al: Adenoviral expression of p53 represses telomerase activity through down-regulation of human telomerase reverse transcriptase transcription. Clin Cancer Res 6: 1239-1247, 2000.

18. Wirth T, Zender L, Schulte B, et al: A telomerase-dependent conditionally replicating adenovirus for selective treatment of cancer. Cancer Res 63: 3181-3188, 2003.

19. Song JS: Adenovirus-mediated suicide SCLC gene therapy using the increased activity of the hTERT promoter by the MMRE and SV40 enhancer. Biosci Biotechnol Biochem 69: 56-62, 2005.

20. Abdul-Ghani R, Ohana P, Matouk I, et al: Use of transcriptional regulatory sequences of telomerase (hTER and hTERT) for selective killing of cancer cells. Mol Ther 2: 539-544, 2000.

21. Liu J, Zou WG, Lang MF, et al: Cancer-specific killing by the $\mathrm{CD}$ suicide gene using the human telomerase reverse transcriptase promoter. Int J Oncol 21: 661-666, 2002.

22. Takakura M, Kyo S, Kanaya T, et al: Cloning of human telomerase catalytic subunit (hTERT) gene promoter and identification of proximal core promoter sequences essential for transcriptional activation in immortalized and cancer cells. Cancer Res 59: 551-557, 1999.

23. Horikawa I, Cable PL, Afshari C and Barrett JC: Cloning and characterization of the promoter region of human telomerase reverse transcriptase gene. Cancer Res 59: 826-830, 1999.

24. Dolph PJ, Huang JT and Schneider RJ: Translation by the adenovirus tripartite leader: elements which determine independence from cap-binding protein complex. J Virol 64: 2669-2677, 1990. 
25. Huang W and Flint SJ: The tripartite leader sequence of subgroup $\mathrm{C}$ adenovirus major late mRNAs can increase the efficiency of mRNA export. J Virol 72: 225-235, 1998.

26. Logan J and Shenk T: Adenovirus tripartite leader sequence enhances translation of mRNAs late after infection. Proc Natl Acad Sci USA 81: 3655-3659, 1984.

27. Lee WW, Lee B, Kim SJ, Jin J, Moon DH and Lee H: Kinetics of iodide uptake and efflux in various human thyroid cancer cells by expressing sodium iodide symporter gene via a recombinant adenovirus. Oncol Rep 10: 845-849, 2003.

28. Shin JH, Yi JK, Lee YJ, et al: Development of artificial chimerical gene regulatory elements specific for cancer gene therapy. Oncol Rep 10: 2063-2069, 2003.

29. Lee WW, Moon DH, Park SY, Jin J, Kim SJ and Lee H: Imaging of adenovirus-mediated expression of human sodium iodide symporter gene by $99 \mathrm{mTcO} 4$ scintigraphy in mice. Nucl Med Biol 31: 31-40, 2004

30. Yoon DW, Lee H, Seol W, De Maria M, Rosenzweig M and Jung JU: Tap: a novel cellular protein that interacts with tip of herpesvirus saimiri and induces lymphocyte aggregation. Immunity 6: 571-582, 1997.

31. Kang HC, Chae JH, Lee YH, et al: Erythroid cell-specific alpha-globin gene regulation by the CP2 transcription factor family. Mol Cell Biol 25: 6005-6020, 2005.
32. Lee H, Kim J, Lee B, et al: Oncolytic potential of E1B $55 \mathrm{kDa}-$ deleted YKL-1 recombinant adenovirus: correlation with p53 functional status. Int J Cancer 88: 454-463, 2000.

33. Komata T, Kondo Y, Kanzawa T, et al: Treatment of malignant glioma cells with the transfer of constitutively active caspase- 6 using the human telomerase catalytic subunit (human telomerase reverse transcriptase) gene promoter. Cancer Res 61: 5796-5802, 2001

34. Cerni C: Telomeres, telomerase and myc. An update. Mutat Res 462: 31-47, 2000.

35. Wang J, Xie LY, Allan S, Beach D and Hannon GJ: Myc activates telomerase. Genes Dev 12: 1769-1774, 1998.

36. Kim E, Kim JH, Shin HY, et al: Ad-mTERT-delta19, a conditional replication-competent adenovirus driven by the human telomerase promoter, selectively replicates in and elicits cytopathic effect in a cancer cell-specific manner. Hum Gene Ther 14: 1415-1428, 2003.

37. Su CQ, Sham J, Xue HB, et al: Potent antitumoral efficacy of a novel replicative adenovirus CNHK300 targeting telomerasepositive cancer cells. J Cancer Res Clin Oncol 130: 591-603, 2004. 